\title{
Role of the response in associative interference
}

\author{
G. ROBERT GRICE, LYN CANHAM, and CHARLES SCHAFER \\ University of New Mexico, Albuquerque, New Mexico 87131
}

\begin{abstract}
Two letter identification experiments were conducted using identical, irrelevant, or responseincompatible noise letters flanking the target in the display. The task was disjunctive reaction time (DRT), in which the subject responds to one target but not the other. Significant associative interference due to response-incompatible noise was not obtained. The effect is consistently obtained in comparable choice reaction time (CRT) experiments. It is concluded that this form of interference depends upon the presence of an active competing response. A CRT experiment was also conducted with a keypress and a verbal response as the competing responses. It produced the same interference as an experiment with two keypresses, suggesting that the dimension of response discriminability is not a factor in determining the amount of interference.
\end{abstract}

When the target letter in a letter identification task is flanked by a noise letter associated with a competing response, choice reaction time (CRT) is increased relative to conditions in which the noise letter is the same as the target or irrelevant to the task (Eriksen \& Eriksen, 1974; Eriksen \& Schultz, 1979). Grice, Canham, and Schafer (in press), applying the methods of variablecriterion theory to the RT distributions, have investigated the temporal dynamics of interference in this situation. The increased RT produced by the irrelevant noise letter was attributed to a form of perceptual interference. This effect occurred very early and merely lowered the entire function for the growth of excitatory strength without affecting its shape. Responseincompatible noise also produces perceptual interference, but, in addition, produces associative interference. The effect of associative interference was nonmonotonic. Completely absent at short latencies, associative interference gradually increased to a maximum at about $450 \mathrm{msec}$ and then gradually decreased to zero at long latencies.

Grice et al. (in press) proposed one tentative explanation of the nonmonotonic effect. As both responses grow in strength, the subject may actively inhibit errors. This process may also delay the correct response, either by the use of limited capacity or by spread of the inhibition to both responses. If inhibition of the error is successful, the correct response will occur later, accounting for the period of recovery from associative interference. An implication of this interpretation is that, if the response-incompatible letter were associated with withholding response rather than with an active response, interference should be eliminated or greatly reduced. This possibility is investigated here by using the disjunctive reaction time (DRT) procedure, or $\mathrm{c}$ reaction,

This research was supported by PHS Grant MH16400 from the National Institute of Mental Health. Requests for reprints should be sent to G. Robert Grice, Department of Psychology, University of New Mexico, Albuquerque, New Mexico 87131. rather than CRT. The results are compared with those of CRT experiments, using the same stimuli, previously reported by Grice et al. (in press).

\section{EXPERIMENTS 1 AND 2}

\section{Method}

Procedure. The experimental situation and general procedures were the same as those previously described in detail by Grice et al. (in press). The target letter appeared in the middle of a video monitor directly above a plus sign used as a fixation point. The target was flanked on both sides by the same noise letter. The noise letter was identical to the target, irrelevant to the task, or the other target letter (response incompatible). The three letters appeared and the fixation point went off simultaneously. The target letters were $A$ and $H$, and the irrelevant noise letter was $K$. The response was pressing a telegraph key with the index finger of the preferred hand. Subjects were instructed to respond to one of the targets but not to respond to the other. A was the positive target for one half of the subjects and $\mathrm{H}$ for the other half. This counterbalancing produced no significant effects. The experiment began with 50 practice trials followed by a short rest. There were then 250 trials without interruption. Data are based on the final 240 trials. Each target was presented equally of ten and equally often with each noise condition. For the final 240 trials, there were 40 trials of each noise condition with each response requirement. Each trial was preceded by an auditory warning signal by an interval of 1.0 , $1.2,1.5,1.8$, or $2.0 \mathrm{sec}$. Trials were in an irregular order, and the interval between trials was $4 \mathrm{sec}$. Stimuli were terminated by the response or after $1.6 \mathrm{sec}$ if there was no response.

Two experiments were conducted: one with accuracy instructions and one with a deadline contingency. The deadline, determined separately for each subject, was the mean RT of Practice Trials 21-50. Feedback was given for errors in both experiments and for failure to meet the deadline in Trials 51-300 in the deadline experiment.

Subjects. The subjects were undergraduate women from courses in introductory psychology. They received course credit for participation. There were 28 subjects in each experiment, including the two CRT experiments presented for comparison.

\section{Results}

Mean and SD RTs are presented in Table 1. The associative interference effect is indicated by compari- 
Table 1

Means and Standard Deviations (SD) in Milliseconds of Mean Correct RT for the Two DRT Experiments and the Two Comparable CRT Experiments

\begin{tabular}{|c|c|c|c|c|c|c|}
\hline & \multicolumn{2}{|c|}{$\begin{array}{c}\text { Identical } \\
\text { Noise }\end{array}$} & \multicolumn{2}{|c|}{$\begin{array}{l}\text { Irrelevant } \\
\text { Noise }\end{array}$} & \multicolumn{2}{|c|}{$\begin{array}{c}\text { Incompatible } \\
\text { Noise }\end{array}$} \\
\hline & Mean & SD & Mean & SD & Mean & SD \\
\hline & \multicolumn{6}{|c|}{ Accuracy } \\
\hline \multirow[t]{2}{*}{ CRT } & $\begin{array}{l}418.2 \\
449.6\end{array}$ & $\begin{array}{l}88.1 \\
58.6\end{array}$ & $\begin{array}{l}427.1 \\
468.4\end{array}$ & $\begin{array}{l}90.2 \\
57.5\end{array}$ & $\begin{array}{l}431.7 \\
488.7\end{array}$ & $\begin{array}{l}91.8 \\
59.7\end{array}$ \\
\hline & \multicolumn{6}{|c|}{ Deadline } \\
\hline $\begin{array}{l}\text { DRT } \\
\text { CRT }\end{array}$ & $\begin{array}{l}338.4 \\
394.2\end{array}$ & $\begin{array}{l}37.8 \\
39.3\end{array}$ & $\begin{array}{l}348.3 \\
412.6\end{array}$ & $\begin{array}{l}34.6 \\
37.9\end{array}$ & $\begin{array}{l}350.8 \\
432.1\end{array}$ & $\begin{array}{l}38.0 \\
40.1\end{array}$ \\
\hline
\end{tabular}

sons of irrelevant and incompatible noise, and perceptual interference is indicated by comparison of identical and irrelevant noise. As reported by Grice et al. (in press), both effects were significant in the CRT experiments. In the present DRT experiments, however, the associative interference effect was essentially absent. With an average difference of only $3.55 \mathrm{msec}$ between irrelevant and incompatible noise, the effect was not significant $[F(1,54)=2.70$, n.s. $]$. Also, it was not significant for either DRT experiment separately, and the interaction of the speed-accuracy factor with noise type was not significant $[F(1,54)<1)$. The negligible associative interference effect obtained with DRT was significantly less than that obtained with CRT. In a comparison of all four experiments, the interaction of Response Requirements by Noise Type was significant $[\mathrm{F}(1,108)=24.21, \mathrm{p}<.001]$. The three-way interaction, including the speed-accuracy factor, was not significant $[F(1,108)<1]$. These experiments clearly establish that the presence of an active competing response is of major importance in producing the associative interference effect on RT.

As would be expected, the perceptual interference effect, inferred from the comparison of identical and irrelevant noise, was significant in the DRT experiments $[F(1,54)=14.21, p<.001]$. The interaction of Speed-Accuracy by Noise Type was not significant $[\mathrm{F}(1,54)<1]$. However, the effect was smaller than that in the CRT experiments. The interaction of Response Requirements by Noise Type was significant $[F(1,108)=$ $8.28, \mathrm{p}<.01]$. Why this should be true is not clear. It may be related in some way to the absence of the associative interference effect in DRT and the easier task as a whole.

Because of the relatively small number of trials per subject in each condition, a full analysis in terms of variable-criterion theory was not made for the DRT experiments. However, functions for the growth of excitatory strength, based on the group distributions, were plotted and examined. In both experiments, the functions for irrelevant and incompatible noise were indistinguishable and there was no trace of the non- monotonic interference effect found for CRT (Grice et al., in press).

In the DRT experiments, error rates (false alarms) for identical, irrelevant, and incompatible noise were .004, .013 , and .039, respectively, for the accuracy experiment and $.029, .038$, and .117 , respectively, for the deadline experiment. The corresponding error rates for the CRT experiments were .014, .017, and .029 and $.021, .034$, and .097 for the accuracy and deadline experiments, respectively.

\section{EXPERIMENT 3}

The following experiment is based on a suggestion made by Neisser (Note 1), following an informal report of the preceding data. His suggestion was, in effect, that both the keypress and withholding response could be regarded as forms of responding, but forms that are highly discriminable. He then raised the question as to whether the associative interference effect would be obtained in CRT with two active, but highly discriminable, responses such as a keypress and a verbal response. Experiment 3 is an implementation of his suggestion.

\section{Method}

Procedure. Procedures were the same as in the DRT accuracy experiment, except that subjects were asked to press the key for one of the targets and to name the other one. The verbal RT was recorded with a voice key (Grice, 1981). Half of the subjects made the verbal response to $A$ and half to $H$.

Subjects. There were 28 female subjects from the same population used for Experiments 1 and 2.

\section{Results}

Means and SDs of the RTs are presented in Table 2. Data are presented separately for the manual and verbal responses and for both responses combined. In the comparison of irrelevant and incompatible noise, the associative interference effect was significant $[F(1,26)=$ $26.03, p<.001]$. It was also significant for each response separately [for manual, $t(27)=4.186, p<.001$; for verbal, $t(27)=2.442, p<.05]$. The interference effect was larger for the manual response than for the verbal response. However, the Response by Noise Type interaction reached only borderline significance $[\mathrm{F}(1,26)=3.63, .05<\mathrm{p}<.10]$. Verbal responses were slower than manual responses $[F(1,26)=42.78$, $\mathrm{p}<.001]$.

Table 2

Means and Standard Deviations (SD) in Milliseconds of Mean Correct RT in Experiment 3

\begin{tabular}{ccccccccc}
\hline & \multicolumn{2}{c}{$\begin{array}{c}\text { Identical } \\
\text { Noise }\end{array}$} & & \multicolumn{2}{c}{$\begin{array}{c}\text { Irrelevant } \\
\text { Noise }\end{array}$} & & \multicolumn{2}{c}{$\begin{array}{c}\text { Incompatible } \\
\text { Noise }\end{array}$} \\
\cline { 2 - 3 } Responses & Mean & SD & & Mean & SD & & Mean & SD \\
\hline Manual & 473.4 & 57.1 & & 494.6 & 51.5 & & 516.2 & 57.5 \\
Verbal & 535.3 & 76.9 & & 555.5 & 73.6 & & 565.5 & 73.6 \\
Both & 504.3 & 64.1 & & 525.0 & 59.5 & & 540.8 & 61.1 \\
\hline
\end{tabular}


In comparing the associative interference effect with DRT and the conventional CRT experiments, comparisons should be on the basis of the same kind of response (i.e., the manual keypress). For the CRT accuracy experiment, the differences between irrelevant and incompatible noise were about the same, and the Competing Response by Noise Type interaction was not significant $[F(1,54)<1]$. On the other hand, the effect was significantly greater than in the DRT accuracy experiment, and the interaction was significant $[F(1,54)$ $=7.97, \mathrm{p}<.01]$.

The perceptual interference effect was about the same for the verbal and manual response, and this interaction was not significant $[F(1,26)<1]$. It also did not differ significantly from the conventional CRT experiment, but it was significantly greater than in the DRT experiment.

Error rates in Experiment 3 for identical, irrelevant, and incompatible noise were $.010, .009$, and .021 .

\section{DISCUSSION}

An active competing response is of major importance and possibly essential in producing the kind of associative interference resulting from response-incompatible noise in a visual display. A statistically significant interference effect was not found in either of the two DRT experiments. The findings confirm the prediction of the hypothesis that this interference is based on the inhibition of a competing response. If the alternative response mode is not responding, there is nothing to inhibit. Although the prediction was confirmed and the credibility of the inhibition hypothesis was somewhat increased, its truth has certainly not been established. Alternative interpretations still remain. However, any theory of this form of interference must take into account the central role of an active competing response.

Experiment 3 demonstrated equal interference with a keypress response by a letter associated with another keypress response or by a letter associated with a verbal naming response. If these responses represent a large difference on a dimension of response discriminability, as they appear to do, the result implies that this dimension is not involved in determining the amount of interference. This seems to leave the presence or absence of an active competing response as the relevant variable.

\section{REFERENCE NOTE}

1. Neisser, U. Personal communication, 1981.

\section{REFERENCES}

Eriksen, B. A., \& Eriksen, C. W. Effects of noise letters upon the identification of a target letter in a nonsearch task. Perception \& Psychophysics, 1974, 16, 143-149.

Eriksen, C. W., \& Schultz, D. W. Information processing in visual search: A continuous flow conception and experimental results. Perception \& Psychophysics, 1979, 25, 249-263.

Grice, G. R. Accurate reaction time research with the TRS-80 microcomputer. Behavior Research Methods \& Instrumentation, 1981, 13, 674-676.

Grice, G. R., Canham, L., \& Schafer, C. Development of associative and perceptual interference. Perception \& Psychophysics, in press.

(Received for publication August 16, 1982.) 